to the earlier formula, derived by Schwedyar, for the correction of torsion balance readings.

W. Domzalski (Great Britain) reported a series of gravity measurements made in underground workings, readings being obtained also at points on the surface vertically above those below ground-level. By averaging the rock density values measured in the laboratory, he has obtained good agreement with those calculated from the gravity measurements using the standard value for the mean earth density. A more academic note was struck by S. K. Runcorn (Great Britain), who discussed the recent investigations in Great Britain of the direction of magnet ization of rocks, with special reference to sedimentary rocks. Their relevance to theories of continental movements was described, and he emphasized the importance of further measurements to give positive evidence on polar drift and on the relative movements of land masses.

The growing use of geophysical methods in mining operations was typified by a description of a gravity survey in east Carmarthenshire by J. T. Whetton, J. O. Myers and I. J. Watson (Great Britain). 'This detailed survey around a new colliery site, in an area of complex geological structure, has indicated the presence of various faults as well as anticlinal and synclinal structures. These results should help in the planning of the mine-workings, and it will be interesting to see how closely the present geophysical interpretation agrees with the true geological picture. In a second report on a survey in northern Italy, C. Monnet (Italy) dealt with the extended application of the vertical gravity-gradient method. This has been used with excellent results over the areas of interest, and its use as a quantitative interpretation tool in other similar areas was recommended.

An interesting example of international co-operation was afforded by maps, presented by J. W. de Bruyn (Holland), showing the Bouguer and isostatic isogals over most of Europe and North Africa. These maps are on a $1: 5,000,000$ scale with 5- and 25-milligal intervals, and the Bouguer map clearly illustrates the principle of isostasy. The isostatic isogals, based on the Airy-Heiskanen system, show predominantly positive anomalies, and the features of this map were discussed in some detail. A later edition of these maps will be prepared to include a considerable amount of data received recently, and any further information which may become available will be most welcome. H. Closs (Germany) has prepared, in a similar way, a map showing the magnetic $Z$-anomalies over Europe. He hoped to make further progress with this, although there were difficulties in combining the results of different field surveys owing to the lack of a common standard of measurement; in the discussion, some doubts were cast on the practical value of such a map. An analogous seismic-velocity map was suggested; but this would clearly require agreement on the aim of such a map and the choice of one of the various possible types of information to be shown.

A paper by V. Baranov (France) provided three charts to help in the interpretation of magnetic surveys. Plotting the observed results and visual fitting to the normalized values on the charts give a rapid interpretation of the observed anomaly. As an example of the use of the charts, he described a mining survey in the Pyrenees where one ore-body, and possibly a second, had been successfully located. The various geophysical well-logging techniques are in widespread use, and J. L. Mathieu and C. Rosoff
(France) gave a gereral account of the methods currently employed, though omitting the continuous velocity logger now available in America, but not as yet in Europe. 'They described how the original electrical methods, though successful in soft formations, had proved unsatisfactory in hard sandstones and limestones, and they showed how the methods developed since the Second World War had considerably improved the value of well logging in such hard formations. For surface electrical resistivity measurements, O. Koefoed (Holland) proposed a modified method of calculating standard intorpretation curves and gave a set of such curves for a three-layer problem. He critically reviewed Hummel's method of interpretation and suggested an amendment to extend its useful range.

Finally, in a paper particularly appropriate to a meeting at 'The Hague, A. Volker and E. O. Houtsma (Holland) described resistivity measurements made in and near the Zuider Zee to determine the salinity of the subsoil. For the measurements in open water, a special cable had been used which provided nine different electrode combinations for each position on the sea-bed, with a consequent increase in the speed of working. The results are of importance both for agriculture and for the provision of water supplies, a further example of the widespread applications of geophysical surveys.

A. T. Dennison

1 Germain-Jones, D. T., Geophys. Prospecting, 2, 177 (1954).

${ }^{2}$ Vajk, R., Geophys., 19, 237 (1954).

\section{THEORY OF PLASTICITY}

$T$

HE James Clayton Lecture for 1955 of the Institution of Mechanical Engineers was given by Dr. William Prager, who chose as his subject "The Theory of Plasticity: a Survey of Recent Achievements", and Dr. Prager gives an account, both authoritative and entertaining, of the present position of the plastic theory. After a brief historical review, he states carefully the reasons for adopting simplified mathematical models, and emphasizes that the solutions derived therefrom can only be approximations to the physical truth. He then illustrates the stress-strain relations corresponding to the various simplified theories by reference to the displacements of cleverly devised kinematic models. These models are certainly most helpful as means of conveying a clear conception of the discontinuous stress-strain relations characteristic of the plastic state. Dr. Prager uses them for showing the difference between stress-strain theories based on total and incremental strain, a discussion which reveals clearly the conceptual superiority of the incremental laws.

The application of general plastic theorems in the calculation of ultimate load-carrying capacities in the fields of structural and mechanical engineering is then discussed. Methods of obtaining lower and upper bounds on collapse loads are introduced by reference to a simple structural example, in which plasticity takes the form of deforming hinges in a member subjected to a transverse load. The extension of these methods to continuous borlies, with application to mechanical engineering problems, is then described, and a number of illustrations are given. It is found that, while it is comparatively easy to obtain upper bounds in plane and three-dimensional problems, lower bounds are much more difficult to 
realize. Recent advances in the derivation of exact values for critical loads are discussed.

The application of plasticity theory to steady-flow problems in which large plastic deformations occur, although stress and velocity fields do not change with time, is illustrated by reference to the plane problem of sheet extrusion. Another class of problem involving large plastic deformations arises when the shape of the plastically deforming part of a body remains constant, while the size increases as more and more of the originally rigid material becomes plastic. The lecture ends with a short section dealing with dynamic problems, and quotes as an example the case of a beam subjected to a transverse uniformly distributed blast load. The energy given to the beam and stored momentarily as kinetic energy is dissipated in deformation at plastic hinges, which may move progressively along the beam, thus producing plastic deformation over considerable lengths.

Dr. Prager's lecture certainly reveals a remarkable advance in the development of the theory of plasticity in recent years. The large part played by mathematical intuition in the derivation of many of the solutions is evident, and the many cases in which this intuition is successful in achieving solutions to practical problems represent the most stimulating feature of the theory of plasticity for the engineer. As progress continues, the place of intuition declines and systematic analysis takes its place. This survey has been made at a time when the second.stage is well under way in many branches of the theory-a period full of danger as well as promise. The danger is that mathematical models found successful for certain problems may be applied indiscriminately under conditions where they become quite inadequate. This is revealed most clearly at the end of the lecture in the section dealing with dynamic loading, where a factor of over-riding importance, namely, the effect of rate of strain on the stresses producing the deformation, is scarcely mentioned. The lecture is concerned with the theory of plasticity, of which it is a most outstanding and opportune summary. It is for the engineer who applies the theories to satisfy himself that the mathematical model on which the theory is based is appropriate to his problem, either by fundamental investigations or by carrying out suitable tests under practical conditions. M. R. HORNE

\section{CARNEGIE INSTITUTION OF WASHINGTON}

\section{REPORT FOR 1953-54}

T HE report of the president of the Carnegie Institution of Washington, Dr. Vannevar Bush, for the year ended June 30, 1954*, explains in some detail the features of the new retirement plan which came into operation on July 1, 1954. He discusses the principles on which it is based, notably the importance of reaching understanding with those affected at the outset, and securing individual assent in advance to whatever changes or modifications are seen from time to time to be desirable to serve the best interests of the group concerned and provide a balanced. provision against the risks which savings inevitably face. Of the research activities of the Institution,

* Carnegie Institution of Washington. Report of the President for the Year ending June 30,1954 . Pp. 15, (Washington, D.C. : Carnegie Institution of Washington, 1954.)
Dr. Bush notes that fully half the observing time of the 60-in., 100-in. and 200-in. telescopes at the Mount Wilson and Palomar Observatories continues to be devoted to spectroscopic and photometric studies of individual stars. Preliminary results of the spectroscopic investigations of globular cluster stars during the past two years indicate a lower excitation temperature for a given spectral class, and a substantially lower abundance of the metals than is found in population I, while photometric studies have revealed large differences between the two populations in the relation between total luminosity and surface temperature. In co-operation with the National Bureau of Standards, the United States Naval Observatory and the California Institute of Technology, the Institution is examining whether electronic imageconversion techniques offer a means of extending the range of large telescopes or increasing the utility of those of moderate size.

The Department of Terrestrial Magnetism reports considerable progress in our understanding of the structure of the atomic nuclei, based on the observation that most nuclei from fluorine to uranium can be made to radiate characteristic gamma-ray lines when bombarded by relatively low-speed helium ions from an electrostatic generator. About a hundred and fifty energy-states have been detected in some seventy nuclei examined at the Department by this 'Coulomb excitation' process. Experimental observations at the Geophysical Laboratory are gradually providing a quantitative basis for understanding the various assemblages of minerals found in the metamorphic rocks and have led to the synthesis of many minerals found in such rocks, including a series of micas and garnets, the ranges of stability of which as functions of temperature and pressure have been determined. Other studies have demonstrated that the recurrence of proteins in hard parts of recent creatures is widespread and that under favourable conditions fossils may contain amino-acids such as alanine, glycine, valine, leucine, aspartic acid and glutamic acid after 360 million years.

In the Department of Plant Biology, spectral changes of unidentified substances have boen found in illuminated Chlorella suspensions. The work on grass this year has shown that environment may greatly influence the percentage of aberrant or 'offtype' plants that may appear in a stabilized, asexually reproduced strain. Under conditions of intense competition in unfavourable environments, the percentage of sexually produced variants may increase greatly in relation to the normally predominant asexual plants. The absolute absorption spectra of purified chlorophylls $c$ and $d$ and of bacteriochlorophyll have been determined, thus providing a means of determining these pigments in plants which use them for photosynthesis. Studies of forests on both sides of the Pacific suggest that the coast redwood was not a tree of the coast during much of its hundredmillion-year history, but lived at higher elevations in the interior of North America.

Experiments on Salmonella typhimurium in the Department of Genetics indicate that a gene locus occupies a longitudinal section of the chromosome, and that changes occurring in different parts of the locus give rise to different new forms of the gene, that is, alleles. Recent work on the genetic mechanism of bacterial viruses indicates that viral nucleic acid controls viral inheritance, while studies of cell chemistry have focused attention on the importance of the nucleoprotein complex in vital cellular activi- 